\title{
Un paisaje de incertidumbres
}

A landscape of uncertainties

Pablo CidB. I Diego Vallejos 0.

\section{$<$ Resumen>}

Dos oficinas de arquitectura fueron invitadas a reflexionar brevemente en torno al tema de los concursos de arquitectura convocados por el sector público, específicamente aquellos que fueron adjudicados y luego no fueron llevados a cabo. Cómo esto afecta a sus oficinas, la sociedad, la ciudad y a la disciplina en general.

$<$ Abst ract $>$

Two architecture firms were i nvited to di scuss about architectural competitions sumbned by government institutions especially about projects that won and were never built. How does this affect architects, society, the city and the architecture itself.

\section{<Palabras clave>}

CONCURSOS DE ARQUITECTURA, ARQUITECTURA PÚBLICA, BARRIO CÍVICO, PLAZA ITALIA ARQUITECTURA Y SOCIEDAD

\section{$\langle$ Key words>}

ARCHI TECTURAL COMPETI TI ONS, PUBLI C ARCHI TECTURE, CI VI C QUARTER, PLAZA I TALI A, ARCHI TECTURE AND SOCI ETY

\section{Ргеsentación}

Los concursos de arquitectura, se presentan como la oportunidad para que la disciplina se despliegue creativa y competitivamente, en un medio donde no existen todos los incentivos para explorar nuevas formas de habitar y construir (y pensar). Dentro de las reformas del estado contemporáneo, no es ya el sector público la principal fuente de encargos, sin embargo es uno de los grandes convocadores de concursos, en donde la envergadura de sus proyectos permite el desarrollo y la exposición de nuevas ideas de arquitectura.

En nuestro país no se ha desarrollado una costumbre en donde la empresa privada invite concursos abiertos y públicos, y dado que generalmente los encargos de concursos provienen del mismo mandante (el sector público), se crea cierto "estilo" predominante. El campo laboral nacional es reducido (en comparación a la cantidad de arquitectos en preparación) y los pocos concursos abiertos que se presentan en el país, no alcanzan a conformar una vanguardia creativa, que se permita el "lujo" de probar nuevas formas y tendencias, o simplemente de explorar. El espacio para errores no alcanza a crear una masa crítica de propuestas que enriquezcan el discurso arquitectónico. En el ámbito internacional las invitaciones transdisciplinares, públicas y abiertas de concursos a nivel de ideas y anteproyectos, van delineando nuevas fronteras disciplinares. Sería interesante evaluar cómo nuestro país, nuestro campo disciplinar puede desarrollar estos métodos.

Como un escenario de expectativas e incertidumbres, invitamos a 2 oficinas de arquitectos, a reflexionar en torno al tema de los concursos de arquitectura, focalizándonos en una falla del sistema, los concursos ganados y no construidos, en donde se revela la importancia de esta manera de adjudicar la materialización de instituciones, proyectos e ideales. 
Fotografías gentileza de Iglesis-Prat arquitectos

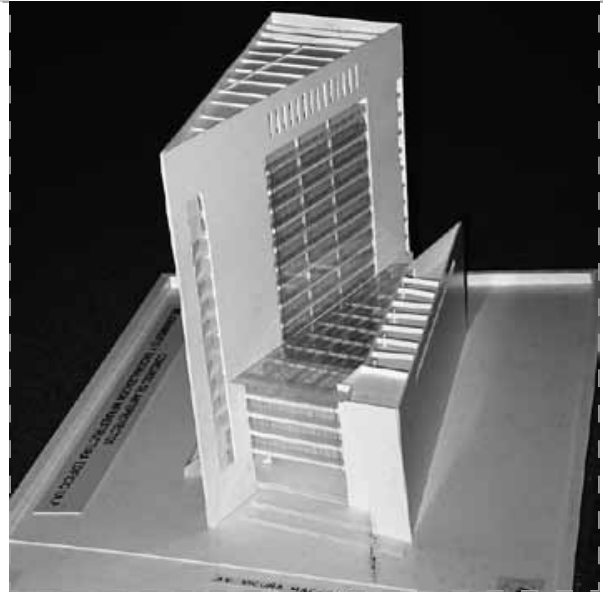

Iglesis-Ргаt Агquitectos

La oportunidad de consolidar proyectos urbanos es una manera de consolidar la cultura de una sociedad: ¿Qué ocurre cuando una sociedad no se hace responsable de materializar sus proyectos e instituciones?

La sociedad en general no comprende el trabajo de los arquitectos. Tampoco lo respeta y valora. No entiende el concepto de calidad de vida asociado a una mejor ciudad. Tampoco los políticos (como autoridades) dimensionan lo significativo de una propuesta urbana integral basada en valores que trascienden del valor de los terrenos y su relación en la gestión inmobiliaria. Los arquitectos no hemos podido hacer ver lo trascendente que resulta enriquecer la cultura a través de un buen proyecto de ciudad.

Los concursos son una oportunidad de posicionamiento para las oficinas más pequeñas dentro del campo disciplinar, que de partida no están siempre invitadas, el riesgo de participar es alto y el antecedente de no construcción de algunos proyectos lo aumenta: ¿Cómo mejorar esta situación? ¿Cómo permitir el posicionamiento de nuevas oficinas de arquitecturas dentro de este sistema?

Los concursos deben ser públicos y abiertos a todos los arquitectos. Deben ser concursos de proyectos no de honorarios ni de ecuaciones extrañas entre bosquejo, currículo y precio. Los concursos se mejoran con un buen director y jurados idóneos. El director hace unas buenas bases, claras, razonables en su alcance, lógicas en su entrega y en sus premios. Verifica además la factibilidad real del encargo por parte del mandante. El jurado debe tener la experiencia y la visión para elegir la mejor solución sin apelar a tablas de valorizaciones parciales ni a silogismos aritméticos de puntaje.

Cuando no se construye un proyecto ganador: ¿Qué perdemos los arquitectos, como gremio, cuando ocurren estos impasses?

Resulta un golpe a los arquitectos, a la arquitectura y a la ciudad. Mucho más allá de lo que significa para el equipo ganador, todos pierden con estas decisiones. Para los arquitectos cada obra es parte de la ciudad y llega a formar parte de ella a través de un concurso público correctamente convocado y jurado. Es garantía de aporte al espacio urbano y a la calidad de vida de todos. Cuando no se construye se mantiene un hoyo negro en medio de la vida de todos.

¿Cómo afecta a una oficina/taller el "proyecto interruptus", la imposibilidad de materializar una obra? Más allá de lo económico: ¿Cómo se afecta el desarrollo del discurso, de la exploración?

La obra que no se construye no tiene ningún valor social ni urbano. Puede que un proyecto señale la vanguardia, sea rupturista o se constituya en referencia, pero a la larga desaparecerá y será olvidado. La historia la hacen las obras así como la vida la constituyen la ciudad y sus edificios.
Proyecto:

Edificio Corporativo Instituto de Normalización Previsional INP

Arquitectos:

Jorge Iglesis Guillard

Leopoldo Prat Vargas

Luis Corvalan Veliz

Colaboradores Arquitectos:

Rossana Pecchi Durand

Mónica Santibáñez Figueroa

Ingeniero Estructural:

Lagos y Contreras SR. René Lagos

Excavaciones y Socalzados:

ISSA KORT

Climatización y Confort Térmico:

Gormaz y Zenteno Ltda. Ingeniería Térmica e Industria

Sistemas de Comunicación

Ingenel Ltda. Sr. José Quintanilla

Instalaciones Eléctricas:

Ingenel Ltda. Sr. José Quintanilla

Instalaciones Sanitarias:

HIDROSUR Sr. Roberto Prat

Seguridad:

IPSA Sr. José Luis Chiuminato

Estudio de Impacto Vial:

CITRA LTDA. Sra. Irene Baeza

Extracción de Basuras:

Duilio Tonini 


\section{No 12}

de arquitectura
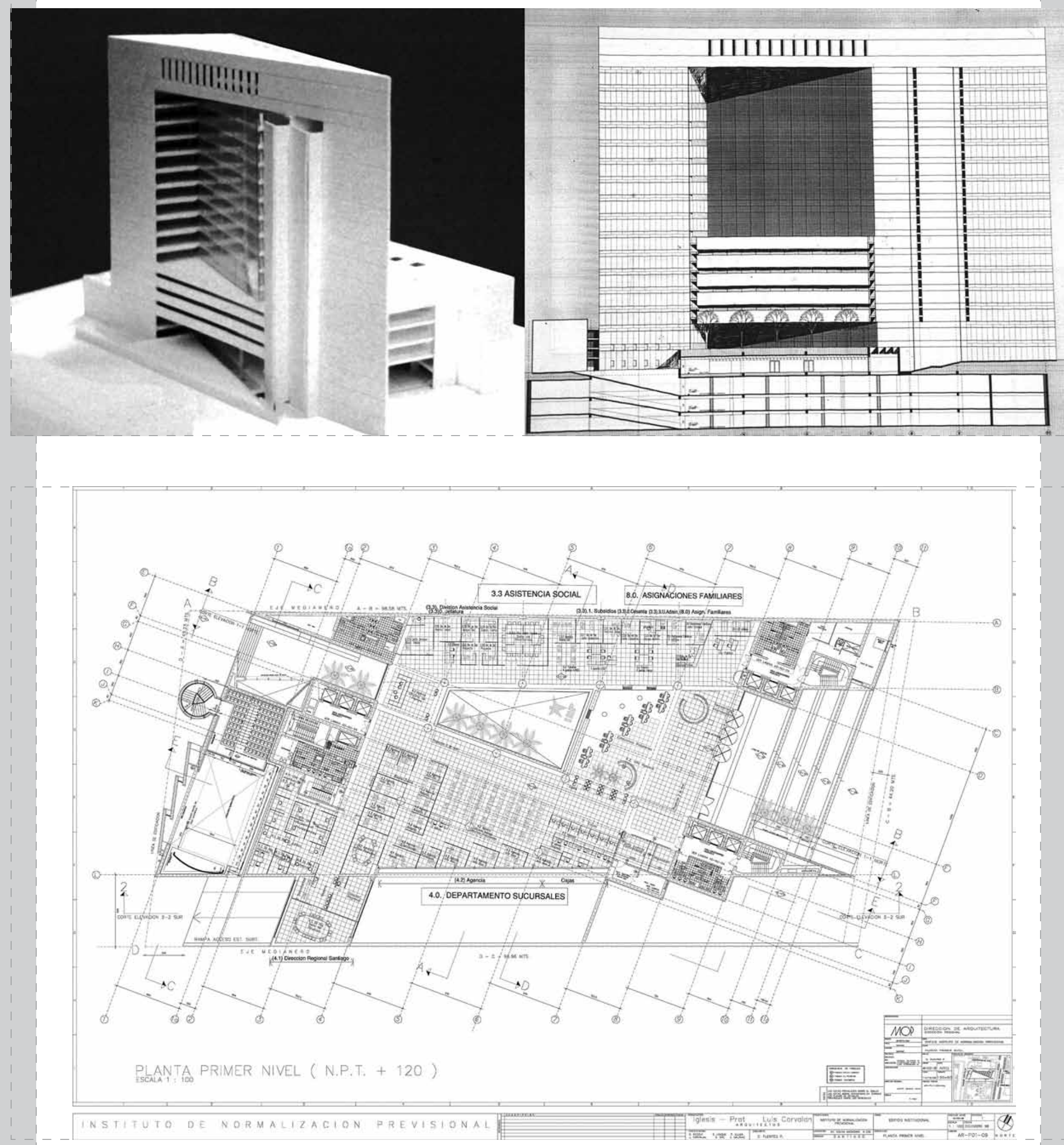


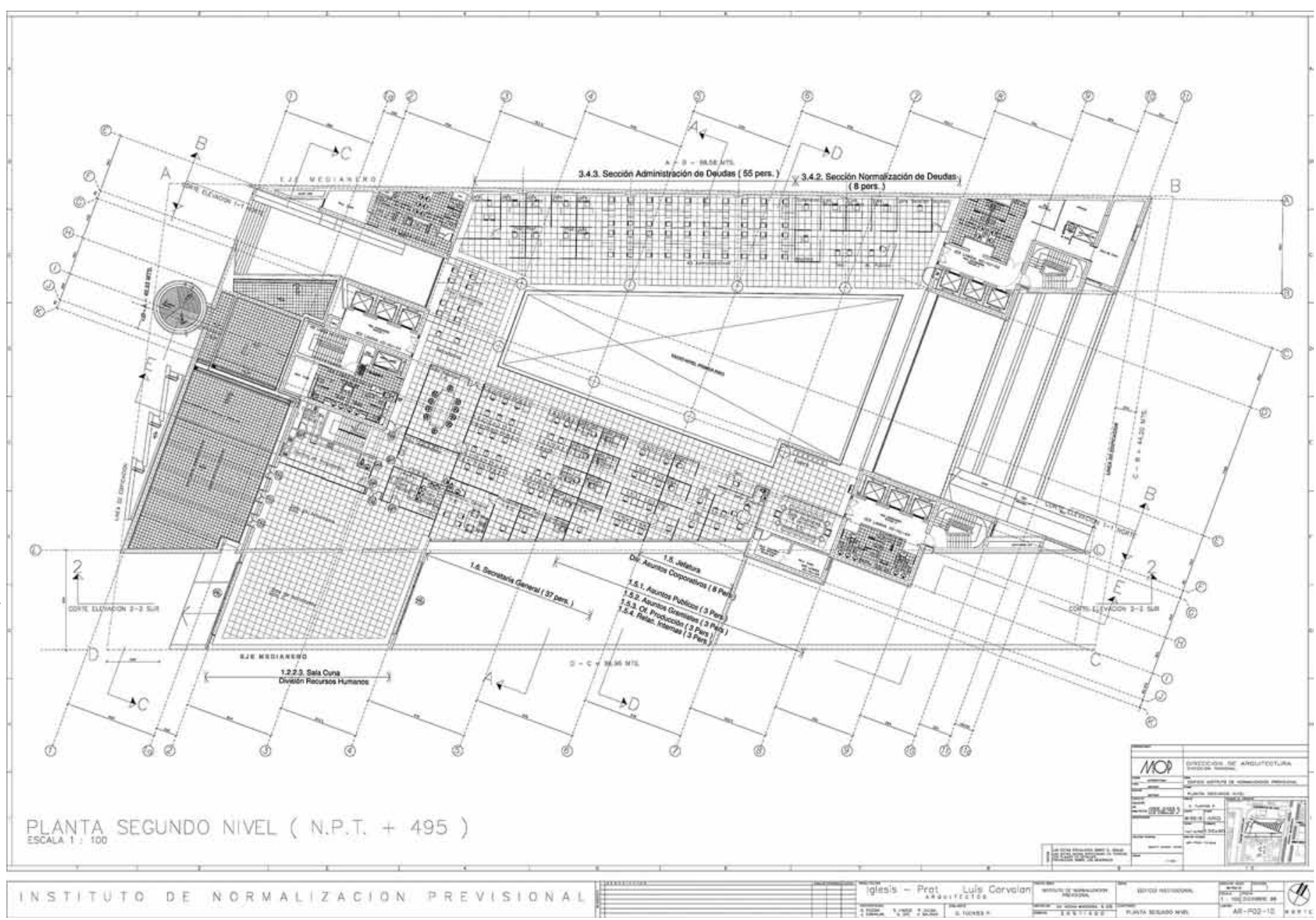

\section{Memoria del proyecto:}

El edificio se implanta en forma diagonal en el terreno enfrentando el Cerro San Cristóbal, orientando su fachada norte al vacío de

la Plaza Italia y a la abertura que ofrece la imponente vista del cerro.

En relación con la imagen corporativa del edificio, la volumetría planteada pretende cumplir con dos objetivos básicos. Por una parte, mostrarse diferente a la tipología de edificio torre San Borja, de carácter habitacional (planta $20 \times 20 \mathrm{~m}$. y 20 pisos de altura) de forma de lograr una identidad propia más allá de la de un edificio de oficinas, y por otra parte, abrirse al público espacialmente, mostrando un edificio amable que invita a su interior público.

La morfología general del edificio sugiere con claridad el acceso principal. La fachada a

Avda. Vicuña Mackenna no es un testero, sino que, en forma oblicua, muestra toda elevación norte del edificio generando el ingreso al hall central.

Esta espacialidad se mantiene en el interior a través de un gran vacío donde se exhiben las plantas más públicas del programa y se abalconan las superiores. Los núcleos de circulaciones verticales, servicios higiénicos e instalaciones, se han dispuestos en la periferia de las plantas liberando el interior de ellas.

La volumetría del conjunto se compone de dos cuerpos. Uno de 7 pisos que hace la transición hacia los edificios del sitio norte y otro de 18 pisos que se levanta como símbolo de poderosa identidad.

El volumen como imagen pública es claro y simple en su forma y materialidad, desprovisto de elementos decorativos, fuera y lejos de modas pasajeras, categórico en su forma de situarse y contemporáneo en el lenguaje arquitectónico. 


\section{Teodoro Fernández}

La oportunidad de consolidar proyectos urbanos es una manera de consolidar la cultura de una sociedad: ¿Qué ocurre cuando una sociedad no se hace responsable de materializar sus proyectos e instituciones?

La sociedad debe primero considerar la ciudad como el espacio donde realiza su vida y se desarrolla, es decir que la calidad de la ciudad está en directa relación con la calidad de vida de la sociedad. Cuando la ciudad se considera principalmente como un espacio mercantil, o por su valor de cambio más que por su valor de uso, los monumentos o el patrimonio pasan a ser más bien una molestia que un beneficio. Sólo cuando un edificio es considerado como obra de arte tiene posibilidades de subsistir en el tiempo y pasar a la categoría de patrimonio. Es en este sentido que la sociedad debe responsabilizarse de construir y cuidar un patrimonio edificado, tanto la ciudad como las instituciones necesitan contar con un patrimonio.

Los concursos son una oportunidad de posicionamiento para las oficinas más pequeñas dentro del campo disciplinar, que de partida no están siempre invitadas, el riesgo de participar es alto y el antecedente de no construcción de algunos proyectos lo aumenta: ¿Cómo mejorar esta situación? ¿Cómo permitir el posicionamiento de nuevas oficinas de arquitecturas dentro de este sistema?

Falta confianza, tanto en las instituciones como en los arquitectos. Los concursos de ideas, con presentaciones más sencillas son una buena posibilidad. Sólo la falta de ideas es mala.

Hay muchas razones para hacer concursos. Sin embargo no debe primar el convencionalismo de los jurados o del mandante.

Cuando no se construye un proyecto ganador: ¿Qué perdemos los arquitectos, como gremio, cuando ocurren estos impasses?

A los arquitectos nos interesa que se construya una ciudad cada vez mejor. Esta es una de las principales razones para participar en concursos, sobre todo si son públicos. Cuando no se construye un proyecto ganador de un concurso público se merma la capacidad del gremio de participar como parte de la sociedad civil, como colaboradores de una sociedad democrática que ha de construirse entre todos.

¿Cómo afecta a una oficina/taller el "proyecto interruptus", la imposibilidad de materializar una obra? Más allá de lo económico: ¿Cómo se afecta el desarrollo del discurso, de la exploración?

El contar con una obra construida es hacer público el pensamiento del arquitecto. No construir es de alguna manera ser marginado de la participación social que el trabajo de cualquier profesión tiene.
Proyecto:

Edificio Ministerio Relaciones Exteriores

Arquitectos:

Teodoro Fenández Larrañaga

Sebastián Hernández Silva

Danilo Martic Vukasovic

Martín Labbé Pinto

Colaborador:

Juan Patricio Poblete Poulsen

Ingeniero Estructural:

Santiago Arias Soto, Ingeniero Civil

Artista Colaborador:

Eduardo Vilches Prieto. Licenciado en Arte, miembro de ACHPE.

\section{Climatización y Confort Térmico:}

Gormaz y Zenteno Ltda. Ingeniería Térmica e Industrial

Sistemas de Comunicación

Alejandro Ríos Marcuello, Ingeniero Civil Eléctrico

Instalaciones Eléctricas:

Proingel Ltda. Proyectos e Instalaciones Eléctricas

Instalaciones Sanitarias:

Sociedad de Ingeniería y Gestión Ambiental, Consultores Ltda.

Estudio de Impacto Vial:

Fernández y De Cea, Ingenieros Consultores. 


\section{Memoria del proyecto:}

El casco histórico de Santiago -el centro-, mantiene su vitalidad y vigencia a partir de una constante renovación y gran diversidad de proyectos que atentos a los diferentes tiempos en que se han construído proponen nuevos modos de uso para este sector de la ciudad. Por otra parte, en los mejores ejemplos de edificación de esta área de la ciudad -el centro-, impone o propone un cierto carácter a sus edificaciones.

La Plaza de La Constitución se construye desde los años treinta en una sucesión de proyectos que apoyándose unos en otros le dan la forma que actualmente tiene. La esquina sur poniente no ha tenido proyectos.
El proyecto para el nuevo edificio del Ministerior de Relaciones Exteriores aquí propuesto trata de concluir en este costado el proyecto para la plaza y el Barrio Cívico: Toma del proyecto para el Barrio Cívico de Carlos Vera la lineación estricta de fachadas y constitución de edificios en correspondencia con la definición de las manzanas y calles. Construye un edificio coincidente con la manzana como los edificios del Ministerio de Hacienda y el Hotel Carrera de Josué Smith. Toma la diagonal de la Plaza de la Constitución y la lleva al interior del nuevo edificio al mismo tiempo que sitúa su acceso como remate de la misma. Alínea la proplongación del edificio de la Contraloría con la línea del palacio de La Moneda. Da a la calle Moneda un portal para los peatones prolongando el caminar por el centro hasta calle A.L.Gotuzzo en correspondencia con la nueva Plaza de La Ciudadanía. Propone un remate superior por detrás de los edificios del Barrio Cívico con la forma de una gran loggia rematada en un parrón volado como modo de ordenar la línea de corte contra el cielo y las ampliaciones sobre estos edificios. Por último, propone un edificio sólido y entonado en colores y materiales respecto al conjunto de los edificios circundantes.

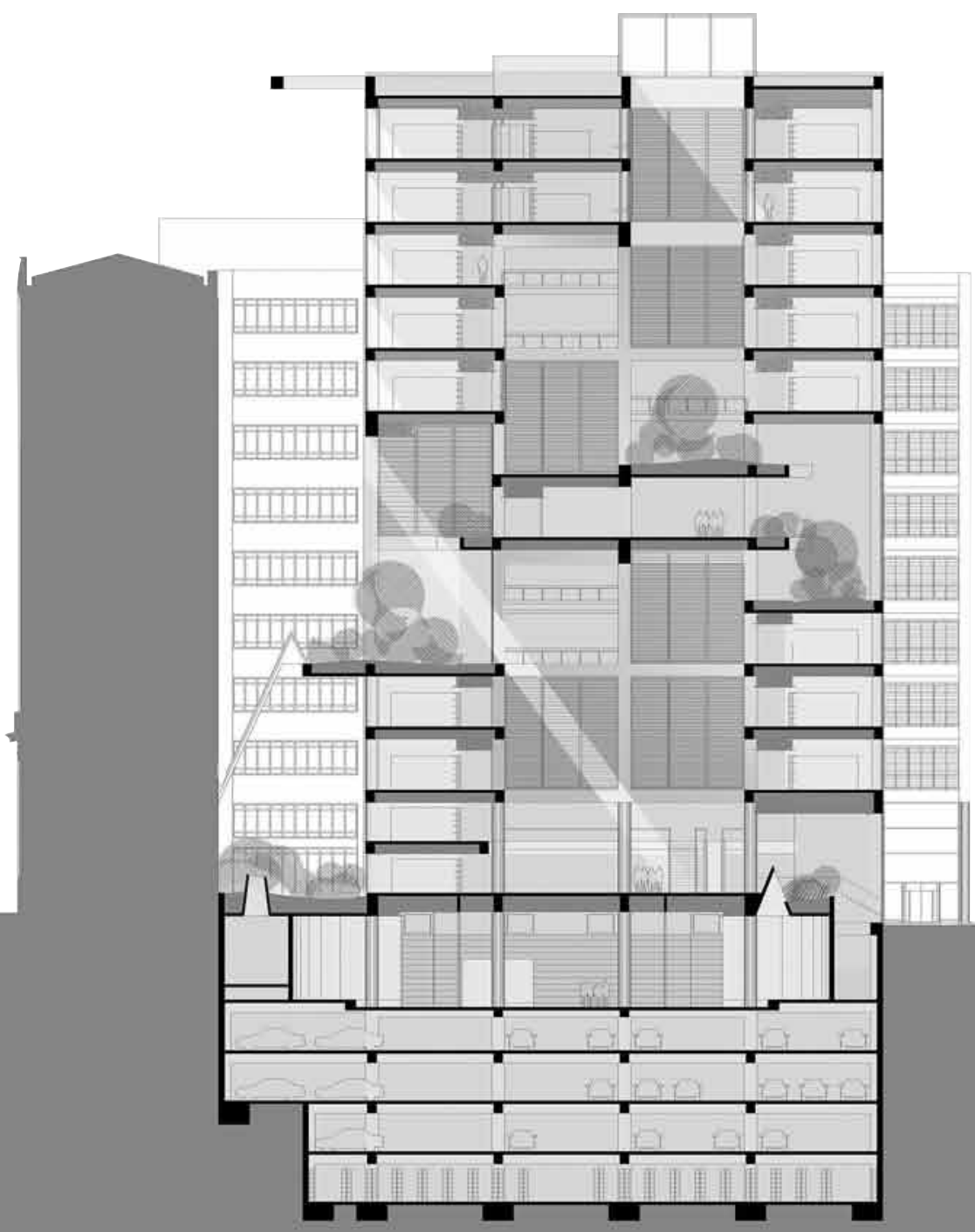



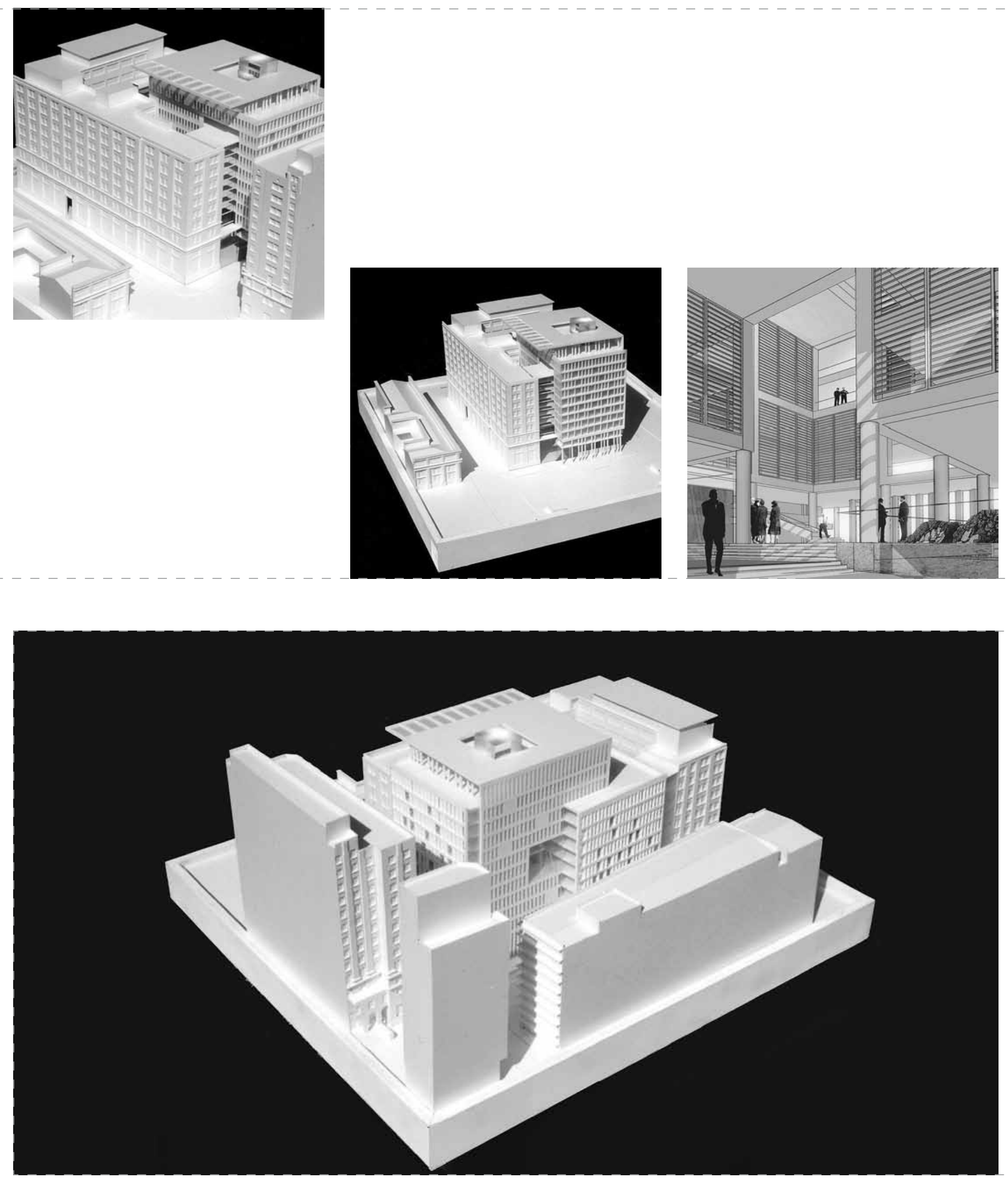

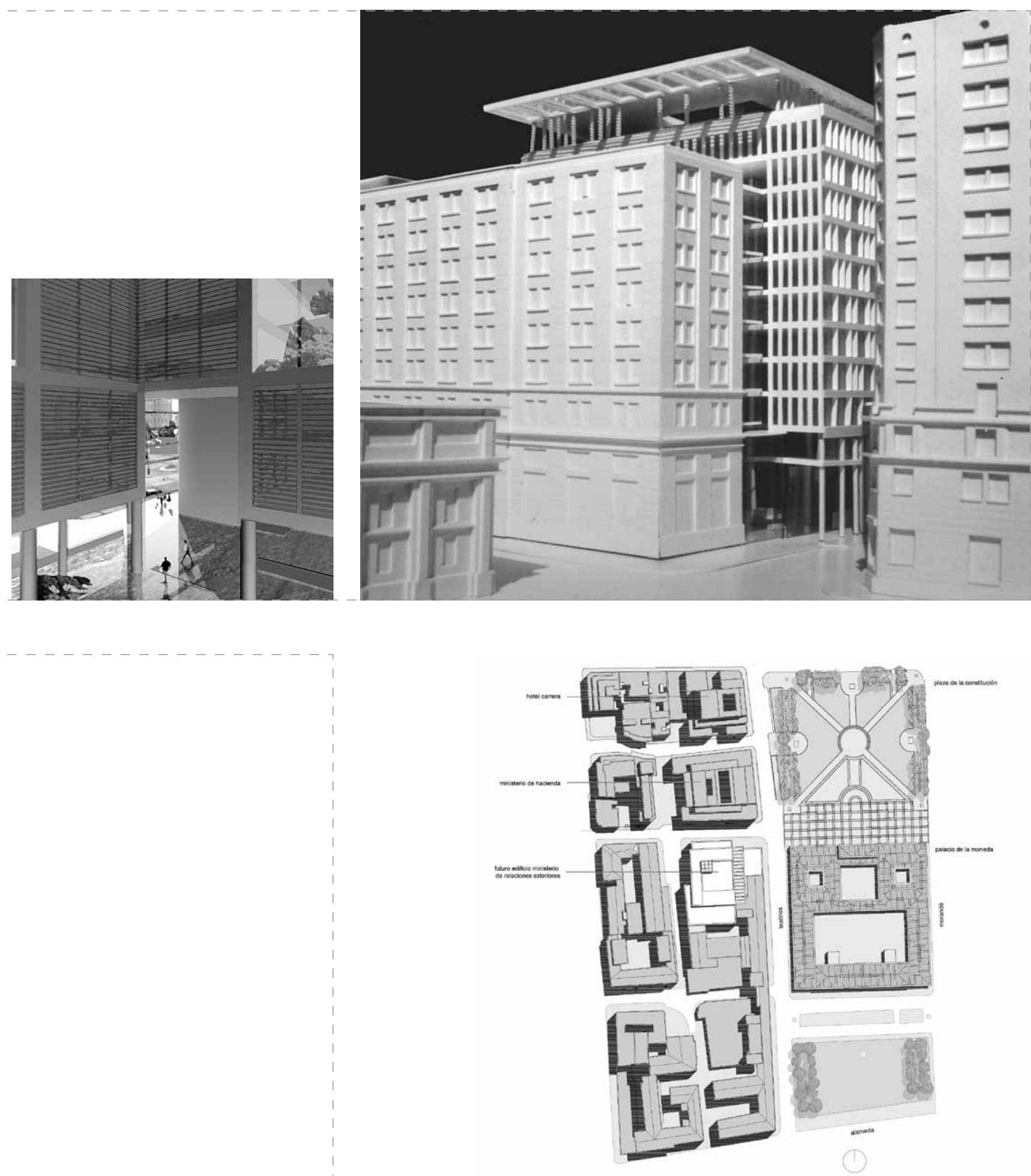